\title{
El papel de la inversión social para garantizar la cobertura del valor de la fuerza de trabajo y su impacto en la productividad: énfasis en los programas del Sistema de Protección Social Universal
}

\author{
Alejandro Salvador Gasteazoro Franco \\ Cecilia Janet Hernández Pleitez \\ Meraris Carolina López Díaz \\ Patricia Alejandra Quintanilla Rivera \\ Universidad Centroamericana "José Simeón Cañas"
}


Furthermore, it is proven (by the historical review of El Salvador's social spend), that social investment increased deeply in 2009 as a result of the creation of the UPSS. Additionally, several global indicators allowed this investigation to prove the negative effects of the workforce's uncoverage.

Finally, the progress and limitations of the UPSS as well as several proposals to improve social investment in El Salvador are exposed.

\section{Palabras clave:}

Economía, El Salvador, teoría económica marxista, programas sociales, fuerza de trabajo, productividad laboral

\section{Keywords:}

Economics, El Salvador, Marxist Economic Theory, Social Programs, Workforce, Labor productivity

\section{Introducción}

En la presente tesis se pretende evidenciar la importancia que tiene la intervención estatal en la economía como implementador de programas sociales y su impacto en el desarrollo de la fuerza de trabajo. Principalmente se abordan aquellos encaminados a completar las necesidades insatisfechas en materia de alimentación, salud y educación.

La investigación se fundamenta en la "Teoría de la cobertura del valor de la fuerza de trabajo", de la corriente marxista. Este cimiento teórico permite demostrar la necesidad de la intervención estatal, dado que la idoneidad de la misma estriba en que considera la fuerza de trabajo como la principal fuerza productiva de la sociedad, y por tanto, esta debe encontrarse en un estado óptimo para poder producir y reproducirse.

Este trabajo de tesis busca poner de manifiesto los impactos negativos que la descobertura genera en la economía, teniendo como premisa que los salarios son incapaces de brindar al trabajador(a) el poder de adquirir los bienes y servicios necesarios para la satisfacción de sus necesidades básicas; surgiendo, bajo este contexto, la importancia de la inversión social por parte del Estado.

En el primer apartado se hace una revisión de los diversos planteamientos que abonan a la teoría central, permitiendo configurar el bagaje teórico y el aparato analítico, que sostienen todas las evidencias y afirmaciones plasmadas a lo largo del documento.

En el segundo capítulo se muestra la evidencia histórica de la falta de cobertura del valor de la fuerza de trabajo. Así como la evolución que ha tenido la inversión social en las áreas de salud y educación desde 1963 hasta 2012, 
y cómo en el último quinquenio las acciones gubernamentales han estado enfocadas a complementar la cobertura del valor de la fuerza de trabajo, detallando los programas sociales en las áreas de salud, educación y alimentación, y el impacto que estos tienen sobre la fuerza laboral.

Seguidamente, se realiza una revisión exhaustiva de estudios que demuestran el impacto que la descobertura de las dimensiones mencionadas genera sobre la productividad, y los consecuentes impactos socioeconómicos que se presentan.

Finalmente, se presentan en el apartado IV, los logros y desafíos de los programas sociales, así como una serie de propuestas y recomendaciones para la mejora de los proyectos de inversión pública en salud y educación, desde el enfoque de la teoría del valor de la fuerza de trabajo.

Con la realización de esta tesis, se espera mostrar la incidencia positiva que tienen este tipo de programas sociales en la economía. Este es un aporte que podría mejorar el rumbo de las políticas públicas, pues, a lo largo de la investigación se corrobora una de las hipótesis principales: los programas sociales lejos de ser un despilfarro, son más bien una inversión a corto y largo plazo, ya que de no asegurarse la reproducción de la fuerza productiva más importante de una sociedad, no se alcanzará la capacidad productiva potencial.

\section{Marco teórico}

\section{La teoría de la fuerza de trabajo y su valor}

\subsection{Teoría marxista del valor}

La teoría marxista del valor nace a partir de la crítica que Karl Marx realizó a la Economía
Política Clásica, su fundamento se encuentra en lo que el autor denominó "El fetichismo de la mercancía", esto es:

(...) atribuir a los productos del trabajo, cualidades que no son inherentes a los mismos... Resulta la cosa más natural del mundo el creer, el pensar y el aceptar que las cosas tienen un valor intrínseco, que el ser valores es algo connatural a los productos del trabajo. Este fenómeno es lo que se conoce como el fetichismo de las mercancías, el cual proviene precisamente de la forma mercancía, de considerar a los productos del trabajo no como simples 
valores de uso, no como simples objetos útiles, sino como mercancías. (Montoya, 2000, p. 52)

Entonces, si el valor de una mercancía no es inherente a esta, ¿de dónde proviene tal valor? Dado que en la teoría marxista una mercancía solo puede ser fruto del trabajo humano, lo que le da el valor a la misma es el trabajo, y es justamente en este punto donde radica la genialidad de Marx, porque evidencia que este posee un doble carácter: concreto y abstracto, siendo lo primero lo que confiere el valor de uso a la mercancía, permitiendo además, la transferencia del trabajo pretérito. Lo segundo (abstracto) por su parte, da lugar al valor, en cuya forma se expresa el valor de cambio.

Es a partir de lo anterior que Marx deriva la teoría de la fuerza de trabajo, y como bien lo expresa Montesino:

El trabajo abstracto es el que genera el valor de cambio de la mercancía, que se expresa en su capacidad de canjearse en determinadas proporciones por otras. Marx dedujo de aquí la Ley del Valor: todas las mercancías se intercambian entre sí de acuerdo a su valor, de acuerdo al trabajo socialmente invertido en su producción. Es a través de esta idea del doble carácter del trabajo que Marx llega a deducir la teoría de la fuerza de trabajo. (Montesino, 2007, p. 828)

La fuerza de trabajo, por tanto, adquiere su valor por la acción que el trabajador ha ejercido sobre ella, a esto se le denominado: el principio del valor-trabajo.

Según Montesino (2011a) este es un principio axiomático, junto a otros 3 constituyen los pilares fundamentales de la teoría económica marxista. El autor realiza un esfuerzo por configurar un aparato axiomático que permite construir un modelo sistemático que a continuación se presenta:

1. Existe una división social del trabajo desarrollada.
2. Solamente el trabajo crea valor. El valor de las mercancías solo lo da el trabajo.

3. El trabajo tiene un doble carácter: trabajo concreto y trabajo abstracto.

4. La racionalidad reproductiva se expresa en términos de valores de uso (bienes) o mercancías necesarias. (Montesino, 2011a, p.p. 26-27)

Tales premisas son básicas en la construcción de la teoría de la fuerza de trabajo y su valor, pues según Montesino (2007), a partir de 
esa sistematización, a Marx le fue posible precisar el concepto de la fuerza de trabajo, la importancia de su valor y cobertura del mismo para el sistema capitalista.

Según Marx, citado por Montesino (2011a) la fuerza de trabajo es definida como "el conjunto de las facultades físicas e intelectuales que existen en el cuerpo de un hombre, en su personalidad viva, y que debe poner en movimiento para producir cosas útiles" (p. 62).

Dado que la fuerza de trabajo es una mercancía especial, su existencia y el aseguramiento de las condiciones necesarias para su reproducción son un pilar fundamental para el sistema capitalista, pues el que la fuerza de trabajo mantenga la calidad normal de sus condiciones físicas y espirituales, significa garantizar su efectiva participación en el proceso productivo.

Al igual que el resto de mercancía, la fuerza de trabajo posee un valor y un valor de uso. El valor de uso es la puesta en acción de las capacidades humanas para la generación del excedente, esto es la plusvalía. Por su parte, el valor es aquella cantidad de bienes $y$ servicios que permiten a la fuerza de trabajo su reproducción.

Según Montesino (2011b), hay dos factores que deben tenerse en cuenta en el establecimiento del valor de la fuerza de trabajo:

1. Valor abstracto de la fuerza de trabajo: tiempo de trabajo socialmente necesario para producirla.

2. Valor de cambio: conjunto de bienes y servicios que la fuerza de trabajo necesita para subsistir; definido específicamente como valor concreto de la fuerza de trabajo.

Montesino (2011b) denomina a la proporción en que se intercambia la fuerza de trabajo por otras mercancías valor concreto de la fuerza de trabajo, el autor manifiesta que tal valor concreto puede ser coherente o no con la forma precio del valor de la fuerza de trabajo, lo que da paso a definir la cobertura del valor de la fuerza de trabajo.

Con respecto a esto último se identifica la segunda diferencia de la fuerza de trabajo frente a las demás mercancías:

...mientras un poseedor de una mercancía que no es fuerza de trabajo, puede por cualquier razón cambiarla por otra por debajo de su valor, sin que ello signifique el deterioro de su nivel de vida (siempre que solo afecte su excedente); en el caso de la fuerza de trabajo la venta de esta mercancía por debajo de su valor deteriora las condiciones 
de vida del trabajador y, por tanto, la calidad normal de la fuerza de trabajo. (Montesino, 2011b, p. 43)

Si la fuerza de trabajo es vendida por debajo de su valor implica que esta será incapaz de cubrir el valor de los medios que le aseguren su existencia, imposibilitando de esta forma un estado normal de vida y trabajo.

En coherencia con lo planteado por Marx citado por Montesino (2011b), sostiene que la economía capitalista está fundamentada en la fuerza de trabajo, la cual es capaz de producir más valor que el que ella misma posee, pero también está asentada en la ampliación de la explotación, siendo su prioridad sacar la mayor diferencia entre el nuevo valor creado y el trabajo pagado.

En este punto es importante mencionar la racionalidad reproductiva, tanto desde el punto de vista de los capitalistas como de los trabajadores.

En este sentido, Montesino (2007) sostiene que:

\begin{abstract}
El sistema históricamente se ha estructurado impulsado por el afán del capitalista de obtener la más alta plusvalía, este comportamiento se expresa en la realidad como una racionalidad medio-fin y costo-beneficio, de modo que mientras el capitalista se las ingenia para obtener la más alta plusvalía como si se tratase de una necesidad vital, el obrero se afana por lograr la completa cobertura del valor de su fuerza de trabajo, esto es, la cobertura de todas sus necesidades humanas. (p. 17)
\end{abstract}

En una sociedad en donde existen dos grupos sociales, uno que solamente posee su fuerza de trabajo y otro que es propietario de los medios de producción, necesariamente hay dos valores relevantes en el proceso económico y social: el valor que expresa las necesidades del trabajador, quien solamente cuenta con su capacidad del trabajo, y el valor que le permite a los capitalistas, grupo poseedor de los medios de producción, obtener la plusvalía, esto es la parte del valor creada por el obrero en la jornada del trabajo de la que el capitalista se apropia como pago por convertir en capital los medios de producción de los cuales son propietarios.

Marx plantea un proceso de reproducción en el cual obreros y capitalistas obtienen lo necesario para vivir humanamente, $y$ a su vez los medios de producción se reponen y se amplían. Una sociedad que opere con este 
sistema de trabajo permite, en condiciones ideales, su reproducción económica y social.

Sin embargo, para Marx ese proceso en la realidad está lejos de ser idílico, debido a que el capitalista posee una racionalidad de la plusvalía. El sistema históricamente se ha estructurado impulsado por el afán del capitalista de obtener la más alta plusvalía posible, lo cual se expresa en la realidad como una racionalidad medio-fin y costo-beneficio. Al mismo tiempo que el capitalista mantiene esta racionalidad los obreros se afanan por lograr la completa cobertura del valor de su fuerza de trabajo, esto es la cobertura de todas sus necesidades humanas.

\subsubsection{Cobertura del valor de la fuerza de trabajo y su efecto en la eficiencia}

Según Montesino (2011a) el deterioro de la fuerza de trabajo no solo repercute en la capacidad productiva del trabajador, sino que a su vez implica un desaprovechamiento tecnológico, evidenciándose en la disminución de la composición orgánica del capital, significando la caída de la tasa de explotación. El deterioro de la fuerza de trabajo puede ser tal, que aunque se den mejoras tecnológicas, estas no podrán compensar la disminución de la productividad o la eficiencia.

El mismo autor afirma que:

...cuando la disminución en la productividad es superior a la disminución de la cobertura del valor de la fuerza de trabajo, la tasa de explotación cae, y si lo hace más rápido que la caída de la composición orgánica la tasa de ganancia también disminuiría, provocando una situación crítica para el sistema económico capitalista. (Montesino, 2011a, p. 51).

El mismo Montesino (2011a) afirma que dada la racionalidad costo-beneficio en la que se basa la economía capitalista, es muy probable que nunca se haya alcanzado la productividad máxima, y por ende, tampoco la producción potencial. En consecuencia lógica, también existe la imperante probabilidad de que la fuerza de trabajo jamás haya sido plenamente cubierta.
Dadas estas premisas se vuelve fundamental para efectos de la presente tesis retomar y actualizar la evidencia empírica existente, de tal manera que pueda demostrarse el papel fundamental que juega el Estado a través de la inversión social, para garantizar la plena cobertura del valor de la fuerza de trabajo, y lo que ello implica para la eficiencia productiva del país. 


\subsection{Otras teorías}

Para el fundamento teórico de la investigación, además de la teoría marxista, también se han considerado otros enfoques que se describen de manera breve a continuación.

\subsubsection{Enfoque de derechos}

De acuerdo al PNUD (2007), los derechos humanos "son garantías jurídicas universales que protegen a los individuos y a los grupos de las acciones y omisiones que interfieren con sus libertades $y$ derechos fundamentales y con la dignidad" (p. 7). La universalidad se garantiza debido a que los derechos civiles, políticos, económicos, sociales y culturales se encuentran dentro de ordenamientos jurídicos internacionales.

Un aporte importante del enfoque basado en derechos humanos consiste en que permite superar la visión simplista que asocia pobreza con la carencia de recursos. Más bien, este enfoque reconoce que la pobreza no se caracteriza únicamente por la ausencia de ingresos, sino también por "la ausencia de bienes físicos y sociales, tales como el empleo, la salud, la integridad física, la protección contra la intimidación y la violencia, la participación en el entorno social, político y cultural; y la posibilidad de llevar una vida con respeto y dignidad" (PNUD, 2007, p. 7)
Es así que el nexo de interdependencia entre pobreza y derechos humanos se debe a que la pobreza es tanto "una causa como una consecuencia del incumplimiento de todas las categorías de los derechos". Es decir, los recursos materiales y bienes físicos y sociales posibilitan el acceso a los derechos, pero también estos facilitan la superación de la pobreza, por ejemplo el derecho a la educación (PNUD, 2007).

\subsubsection{Enfoque del ciclo de vida}

Este enfoque pretende garantizar que se asegure el cumplimiento de los derechos de los individuos desde antes de su nacimiento. En especial garantizando el cumplimiento de los derechos de las mujeres en edad fértil o embarazadas. El objetivo es que las intervenciones en cada etapa de vida garanticen que en la edad adulta los individuos tengan los elementos necesarios para desplegar todo el potencial desarrollado, en especial para ponerlo en práctica en el mercado laboral (PNUD, 2013).

Lo anterior refleja que el interés de este enfoque es anticiparse a las problemáticas sociales. Es por esta razón que este enfoque se encuentra basado en políticas sociales de acompañamiento de acuerdo a cada etapa de vida, de modo que poco a poco se desarrollen las capacidades de los individuos. 


\subsubsection{Enfoque de libertades y capacidades de Amartya Sen}

Sen se refiere a muchas formas en que la libertad posibilita y permite el desarrollo, por lo tanto su abordaje es esencial.

La libertad en la visión de Sen (2000), es la condición fundamental de la existencia humana, y esto es entendido así porque según su teoría la libertad genera las capacidades humanas, el individuo adquiere la capacidad de hacer (o de ser) lo que considera valioso para él. Esto implica que se está haciendo referencia a un concepto de libertad bastante amplio, se concibe en primera instancia como libertad jurídica y política, de contratación, de participación, pero a su vez tales libertades carecerían de importancia si las capacidades del individuo se viesen afectadas por la falta de salud, que además significa una reducida longevidad, por las limitaciones o ausencia de la educación, por una débil nutrición, por una baja renta, entre otros.

Resulta de suma importancia para la presente investigación el aporte teórico desde este enfoque que considera que para que se dé el desarrollo se exige la eliminación de las principales fuentes de privación de libertad, que Sen (2000) concibe como la pobreza y la tiranía, la escasez de oportunidades económicas y las privaciones sociales sistemáticas, el abandono en que pueden encontrarse los servicios públicos, y que de alguna manera da herramientas teóricas para sustentar la imprescindible intervención del Estado que en esta investigación se plantea.

\subsection{Valoración de las teorías abordadas}

Para efectos de la presente tesis resulta fundamental realizar una valoración acerca de los aspectos favorables y desfavorables de las teorías expuestas anteriormente, pues de esta manera se tendrán como base los elementos a retomar en función de las necesidades de la investigación.

Para nuestro interés Sen (2000), sostiene que la libertad posibilita y permite el desarrollo, ya que para él es la misma libertad la que genera las capacidades humanas, sin embargo -y este es un planteamiento de esencial relevancia para el presente estudio-, él considera que tales libertades (jurídicas, políticas, de contratación, de participación, etc.) carecerían de importancia si las capacidades de las personas son afectadas por la falta de salud, una débil nutrición, por una baja renta... en fin, por la falta de cobertura de todas aquellas necesidades que posibilitan el buen desenvolvimiento de una persona.

Este planteamiento resulta similar a lo expuesto en el desarrollo del Marco teórico, ya que 
está fundamentado en la teoría de la cobertura del valor de la fuerza de trabajo, donde dicha cobertura se entiende como la suma de medios de vida suficientes para mantener al trabajador en su estado normal de vida y de trabajo, lo que le asegura la reproducción y el despliegue de sus potencialidades.

Respecto al enfoque de derechos, el planteamiento de Montesino (1998) dicta que los seres humanos podrán desplegar todas sus potencialidades en la medida en que puedan acceder a una canasta de mercado que les garantice una vida digna. Es por esta razón que la visión basada en los derechos humanos tiene una gran importancia y posee cierta complementariedad, pues establece que un nivel de vida digno se asegura únicamente mediante el acceso de una amplia gama de derechos civiles, políticos, económicos, sociales y culturales. Claramente este enfoque supera el enfoque tradicional sobre el abordaje de la pobreza.

Por su parte, el enfoque del ciclo de vida también se puede complementar con la teoría de la cobertura del valor de la fuerza de trabajo. El objetivo del planteamiento del ciclo de vida es que se apliquen estrategias diferenciadas y específicas durante cada etapa de vida. Esto es clave especialmente en un contexto donde existe una reproducción intergeneracional de la pobreza y desigualdad, pues las políticas sociales que son aplicadas a grupos poblacionales desde la edad temprana permiten que las potencialidades de cada individuo se encuentren desarrolladas en toda su magnitud al momento del proceso productivo.

Resulta claro que las teorías y enfoques expuestos servirán de complemento a la teoría principal en la que se fundamenta esta tesis: "Teoría de la cobertura del valor de la fuerza de trabajo". Es así como teniendo como base esta teoría, la presente tesis pretende evidenciar los impactos que la descobertura genera en la eficiencia productiva para el caso de El Salvador, teniendo como premisa que la forma del precio del valor de la fuerza de trabajo, es decir los salarios, son incapaces de brindar al trabajador el poder de adquirir los bienes y servicios necesarios para su reproducción, surgiendo bajo este contexto la importancia de la inversión social por parte del Estado salvadoreño.

De acuerdo con Montesino (2011a) y partiendo de la propia definición de Marx sobre la fuerza de trabajo, el funcionamiento normal de la misma y el correspondiente aprovechamiento tecnológico en el proceso productivo, depende directamente de su cobertura, lo cual sugiere inevitablemente que una cobertura insuficiente impactará negativamente en su desempeño. 
Es por ello que la teoría de la cobertura del valor de la fuerza de trabajo se convierte en el pilar fundamental para el desarrollo de esta investigación. Todo el bagaje teórico y el aparato analítico permitirán analizar, con el complemento de los otros enfoques, cómo la insuficiente cobertura y el consecuente deterioro de la fuerza de trabajo redundan en la eficiencia productiva del país y las consecuencias que de ella se derivan.

\section{Evidencia histórica de las dimensiones del valor de la fuerza de trabajo: alimentación, educación y salud}

Uno de los hechos que hacen evidente la intervención del Estado a través de la inversión social es la brecha que existe entre los ingresos que perciben los hogares y los gastos que estos llevan a cabo para poder cubrir sus necesidades básicas. El papel del Estado, en este caso, sería asegurar que las personas posean los recursos necesarios para poder subsistir.

Son muchas las necesidades que los seres humanos necesitan tener cubiertas para poder desplegar todas sus potencialidades. Sin embargo, para fines de esta investigación se eligieron tres de ellas: alimentación, salud y educación. En esta sección se pretende conocer el nivel de acceso que tiene la población a la satisfacción de estas necesidades, y ante ello justificar el papel del Estado para poder asegurar que dicha población no experimente carencias respecto a ellas.

\subsection{Alimentación}

Dentro de las necesidades fundamentales a ser cubiertas por las personas se encuentra la alimentación. Ello es debido a que la realización de las actividades diarias, el desarrollo físico y mental de las personas, así como los niveles de productividad que estas puedan generar estarán en concordancia con una ingesta mínima de calorías que conforman una alimentación adecuada.

El acceso a una alimentación adecuada estará determinado por la capacidad adquisitiva de las personas. Para el caso de El Salvador, la Constitución de la República establece que los salarios mínimos deben estar en concordancia con el nivel de vida y ajustarse a otros factores, tales como sistemas de remuneración y zonas de trabajo.

Sin embargo, datos de la Dirección General de Estadística y Censos (DIGESTYC) y del Banco Central de Reserva muestran que los salarios reales han tendido a la baja y que la tasa de crecimiento de los mismos está por debajo del crecimiento que ha presentado la inflación. 
Lo anterior tiene repercusiones notorias sobre el acceso que las personas tienen a una alimentación adecuada. La Canasta Básica Alimentaria (CBA) es uno de los instrumentos que permiten determinar lo anterior. Estadísticas de las fuentes mencionadas anteriormente muestran que el "sector comercio y servicios" es el que presenta un mayor poder adquisitivo respecto a esta canasta, mientras que el "sector agrícola" es el que se ubica en último lugar. Sin embargo, respecto al "sector comercio y servicios" en términos absolutos, después de deducido el costo de la CBA, solamente quedan disponibles US\$45.31 para la satisfacción de otras necesidades básicas. Lo anterior muestra que si las personas tienen dificultades para acceder a una alimentación adecuada, dejan de lado la cobertura de otras necesidades importantes, tales como salud, alimentación, vestuario, esparcimiento, entre otras.

Las anteriores conclusiones se deducen al contrastar el poder adquisitivo de las personas con una canasta que hace referencia a una alimentación mínima; sin embargo, de acuerdo al enfoque de derechos, no basta con tener una cantidad mínima de calorías a consumir, sino que la alimentación debe ser suficiente en calidad y en cantidad. En aras de lo anterior es que, para esta investigación, se configuraron tres canastas alimentarias nuevas; una para el área rural, otra para el área urbana y una tercera a nivel nacional. Dichas canastas se construyeron basándose en las necesidades nutricionales de las personas promedio, en sus características físicas, alimentos que fuesen de consumo generalizado de costo accesible, alimentos diversificados y de acuerdo a parámetros del Ministerio de Salud.

Una vez construidas las canastas ya mencionadas, y atendiendo a las características y requisitos que estas deberían tener, se obtuvo que el costo de una alimentación mínima para el área rural, urbana y a nivel nacional es de: US\$124.15, US\$175.4 y US\$239.08 respectivamente.

A partir de lo anterior es posible concluir que las familias salvadoreñas tienen un acceso limitado respecto a la alimentación mínima que necesitan para su subsistencia. Esto dificulta el desarrollo socioeconómico de El Salvador, ya que impacta principalmente en el bienestar de las personas más pobres del país.

Castaneda y Rivera (2010), en un Estudio para el Banco Interamericano de Desarrollo, respaldan esta conclusión, pues encontraron que un estado nutricional óptimo logra mejorar la salud, la formación del capital humano y su productividad, respaldando así la hipótesis de que existe una relación positiva entre una buena nutrición 
y la productividad que las personas puedan generar.

\subsection{Evolución de la inversión social en educación y salud}

Ya se ha dicho que las personas, en tanto seres complejos y multidimensionales, no solo necesitan la alimentación para poder subsistir y reproducirse. Parte de las necesidades básicas que necesitan satisfacer es el tener acceso a la educación y a la salud.

La garantía de estas necesidades no siempre ha formado parte de las prioridades de los países. En el caso de El Salvador no es sino hasta el período entre el 2005 y el 2012 que la inversión social se convierte en uno de los principales rubros sobre los cuales se planifica y ejecuta su presupuesto público.

Con el objetivo de tener un conocimiento claro sobre el tema que se expone en la presente investigación es necesario definir el gasto social. De acuerdo al Diccionario de la Administración Pública de El Salvador, el gasto social se entenderá como: "Las erogaciones públicas destinadas al financiamiento de servicios sociales básicos, que según la clasificación propuesta por la Organización de las Naciones Unidas, son los gastos en educación, salud, seguridad social, vivienda, deportes y otros de características similares» (Ministerio de Hacienda, 2012).

\subsection{Estructura del gasto social en El Salvador}

De acuerdo al Ministerio de Hacienda (2012), quien en su Boletín Presupuestario (Enero-Marzo 2012), sostiene que el gasto social en El Salvador está conformado por las instituciones y entidades públicas que tienen a su cargo la definición y coordinación de políticas en materia de educación, salud pública y asistencia social, trabajo y previsión social, vivienda y desarrollo urbano, así como todos aquellos programas relacionados con el desarrollo local, seguridad social, apoyo al deporte, desarrollo integral de la niñez y adolescencia, etc., cuya responsabilidad no solamente es del gobierno central, sino también de aquellas instituciones descentralizadas vinculadas a este.

De forma más concreta, el gasto social forma parte del "Área de Desarrollo Social" que de acuerdo a la misma fuente, agrupa todos los servicios que presta el gobierno para mejorar la calidad de vida de la población, en especial la de menores ingresos, a efecto de lograr su mejor incorporación al sistema económico y alcanzar su bienestar.

El cuadro de a continuación muestra los componentes del "Área de Desarrollo Social" y los componentes que la conforman: 


\begin{tabular}{|c|c|c|c|c|}
\hline \multicolumn{5}{|c|}{ Gasto Social } \\
\hline \multicolumn{5}{|c|}{ Área de Desarrollo Social } \\
\hline Educación & Salud & Desarrollo Local & Seguridad Social & $\begin{array}{l}\text { Otros servicios } \\
\text { sociales }\end{array}$ \\
\hline $\begin{array}{l}\text { Educación Parvularia, } \\
\text { Básica, No formal } \\
\text { y Superior No } \\
\text { Universitaria. } \\
\text { Instituciones } \\
\text { Adscritas (UES), } \\
\text { Federación } \\
\text { Salvadoreña de } \\
\text { Fútbol, Caja Mutual, } \\
\text { Empleados Ministerio } \\
\text { de Educación. } \\
\text { Consejo Nacional de } \\
\text { la Cultura. } \\
\text { Subsidio, } \\
\text { Instituciones } \\
\text { Educativas y } \\
\text { Culturales. }\end{array}$ & $\begin{array}{l}\text { Sistema Básico } \\
\text { de Salud Integral- } \\
\text { Hospitales } \\
\text { Nacionales. } \\
\text { Instituto Salvadoreño } \\
\text { de Rehabilitación de } \\
\text { Inválidos. } \\
\text { Hogar de Ancianos } \\
\text { "Narcisa Castillo } \\
\text { Santa Ana". } \\
\text { Cruz Roja } \\
\text { Salvadoreña. } \\
\text { Subsidio } \\
\text { Instituciones de } \\
\text { Asistencia Social. }\end{array}$ & $\begin{array}{l}\text { Financiamiento } \\
\text { a Gobiernos } \\
\text { Municipales. } \\
\text { Financiamiento al } \\
\text { Fondo de Inversión } \\
\text { Social para el } \\
\text { Desarrollo Local. }\end{array}$ & $\begin{array}{l}\text { Financiamiento al } \\
\text { Sistema de Pensiones } \\
\text { Público. } \\
\text { Financiamiento } \\
\text { al Programa de } \\
\text { Rehabilitación de } \\
\text { Lisiados. }\end{array}$ & $\begin{array}{l}\text { Trabajo y Previsión } \\
\text { Social. } \\
\text { Vivienda y } \\
\text { Desarrollo Urbano. } \\
\text { Presidencia de la } \\
\text { República (Becas, } \\
\text { Fortalecimiento } \\
\text { Familiar, deportes, } \\
\text { niñez, adolescencia, } \\
\text { desarrollo de la } \\
\text { mujer, subsidios } \\
\text { institucionales, } \\
\text { bienestar social, etc.) }\end{array}$ \\
\hline
\end{tabular}

Fuente: elaboración propia con base en el Boletín Presupuestario del Ministerio de Hacienda, 2012.

\subsection{Educación}

La educación, además de ser un derecho, es una herramienta que permite desarrollar las potencialidades de las personas, ante ello el Estado se encuentran obligado a garantizar el acceso a la misma.

Mediante la ejecución de diferentes acciones, programas y proyectos públicos, el gobierno de El Salvador interviene en la educación para que las personas puedan acceder, de manera que estas no solamente tengan la apertura para adquirir los conocimientos que mejoren su nivel de vida, sino que la educación a la que tengan acceso sea de buena calidad.
Cada uno de los componentes que conforman el área de educación tiene acciones concretas mediantes las cuales es posible alcanzar objetivos específicos respecto a la cobertura, metodología, capacidades de las personas y segmentos de las mismas que el gobierno se ha propuesto alcanzar y mejorar.

\subsection{Presupuesto destinado a salud}

Con el objetivo de garantizar un estado de completo bienestar físico, mental y social, los gobiernos destinan una parte considerable de su presupuesto a actividades, programas y recursos para mejorar la salud, prolongar la vida de las 
personas, mejorarles la calidad de vida, así como prevenir y curar enfermedades.
Para llevar a cabo estos objetivos en El Salvador el gasto social destinado a salud está estructurado de la siguiente manera:

\section{Cuadro 2. Estructura del gasto público destinado a salud}

\begin{tabular}{|l|l|l|l|l|l|l|l|}
\hline \multicolumn{7}{|c|}{ Sector público de salud } \\
\hline \multicolumn{7}{|c|}{ Ministerio de Salud } \\
$\begin{array}{l}\text { Instituto } \\
\text { Salvadoreño } \\
\text { del Seguro } \\
\text { Social. }\end{array}$ & $\begin{array}{l}\text { Instituto } \\
\text { Salvadoreño } \\
\text { del Bienestar } \\
\text { Magisterial. }\end{array}$ & $\begin{array}{l}\text { Comando } \\
\text { de Sanidad }\end{array}$ & $\begin{array}{l}\text { Dilitar. } \\
\text { Nacional de } \\
\text { Medicamentos. }\end{array}$ & $\begin{array}{l}\text { Consejo } \\
\text { Superior } \\
\text { de Salud } \\
\text { Pública. }\end{array}$ & $\begin{array}{l}\text { Otras } \\
\text { entidades } \\
\text { públicas. }\end{array}$ & $\begin{array}{l}\text { Gobiernos } \\
\text { locales. }\end{array}$ & $\begin{array}{l}\text { Entidades } \\
\text { públicas. }\end{array}$ \\
\hline
\end{tabular}

Fuente: elaboración propia con base en estructura presupuestaria del Ministerio de Salud (MINSAL).

\subsection{Análisis de la evolución de la inversión social en salud y educación}

Tras un análisis gráfico y estadístico del comportamiento experimentado de los montos de gasto social que han destinado los gobiernos de El Salvador desde 1963 a 2011, a salud y alimentación, se obtuvieron las siguientes aseveraciones:

1. Tanto el gasto en salud como en educación, de manera general, han presentado una tendencia creciente desde el primer año en análisis hasta el último. El índice del gasto ejecutado en educación y salud mostró que todo el período en estudio tiene una tendencia progresiva, en el sentido de que cada año el Estado destina una mayor cantidad de recursos para el funcionamiento, inversión y en general, para el sostenimiento de estos sectores, y de los distintos programas sociales que en ellos se ejecutan.

2. La inversión social para garantizar la cobertura de ambas necesidades ha aumentado en el período comprendido entre 2005 y 2011, eso era lo que se habían propuesto los gobiernos de este período al fijarse como objetivo profundizar en la inversión social. Para el caso del presupuesto destinado a Salud, este experimentó entre 2005-2012 la tasa de crecimiento más elevada del gasto presupuestado con un $22.57 \%$.

3. El incremento significativo en el presupuesto del gobierno destinado a las áreas de salud y educación en el último período de análisis (2005-2012), es explicado por la implementación del Sistema de Protección Social Universal (SPSU). Fue a mediados del 2009, con el cambio de gobierno, que 
comienzan a experimentarse modificaciones a nivel de políticas sociales, ya que estas últimas comenzaron a planificarse y ejecutarse basándose en los enfoques de derechos y ciclo de vida. Lo anterior se enmarca en las acciones concretas que ha llevado a cabo el gobierno de El Salvador para atender de forma más concreta y directa las necesidades básicas de los salvadoreños y salvadoreñas.

\subsection{Acciones gubernamentales orientadas a subsanar la falta de cobertura del valor de la fuerza de trabajo (2009-2014)}

A partir del año 2009 las acciones de política social Ilevadas a cabo en El Salvador se enmarcan en lo que la UNICEF califica como protección universal. De acuerdo a UNICEF, citado por STPP-UNICEF-ICEFI (2014), este tipo de accionar gubernamental se caracteriza porque tiene la capacidad de prevenir, reducir y eliminar las condiciones que determinan y reproducen la vulnerabilidad a la pobreza.

Con el SPSU la población en mayores condiciones de vulnerabilidad participa en programas que fortalecen sus capacidades y contribuyen en la solución de los problemas sociales, en particular la pobreza, la desigualdad en la distribución de ingresos, desigualdad entre hombres y mujeres, y la exclusión social.

Vale aclarar que la intervención gubernamental no se limita al SPSU, pero se destaca debido a que fue la estrategia más inmediata que se implementó y que incorpora el enfoque de derechos y del ciclo de vida. El gobierno ha intentado complementar el SPSU con las políticas de cada ministerio. En ese sentido, en el quinquenio 20092014 se ha aplicado la primera etapa de un proceso que llevaría a la universalización de los servicios sociales básicos. Esta primera etapa tiene la función de cerrar brechas sociales y de garantizar ciertos servicios de manera universal (paquetes escolares, etc.)

De esta forma, el SPSU se ha enmarcado dentro de otras políticas públicas a largo plazo, complementándose con políticas sociales sectoriales, tales como el Sistema Nacional Integrado de Salud, el Plan Nacional de Educación, la Política de Vivienda y otras políticas transversales como la Política de Seguridad Alimentaria y Nutricional, la Política Nacional de la Mujer, la Política de Juventud (STPP, 2014).

\subsection{Vinculación entre la inversión social y su impacto en los indicadores de bienestar: primera aproximación}

Este apartado permite reflexionar sobre dos cosas: en 
primer lugar, que de la década de los 80 hasta el 2005 aproximadamente, significó un desaprovechamiento de los recursos que se pudieron haber destinado para potenciar la inversión social orientada al desenvolvimiento de las capacidades de las personas. Este comportamiento es atribuible, en un principio, a los cambios de prioridad del Estado, ya que para la década de los 80 , debido a la guerra civil los recursos se destinaban en su mayoría a la Defensa y Seguridad. Por otro lado, la década de los 90 se caracterizó por la búsqueda del crecimiento económico y generación de riqueza, que obedecía a la lógica del modelo neoliberal, dejando de lado la participación del Estado.

Sin embargo, más que analizar las tendencias crecientes o decrecientes de la inversión social, lo que interesa es la compatibilidad que tienen los periodos de más inversión social con el alcance de mejores indicadores de bienestar social y económico. En este sentido, contrario a los periodos anteriores, aproximadamente a partir del 2005 se percibe un aumento significativo de la inversión social en educación y salud, lo central es que estas inversiones se han traducido en resultados importantes.

De acuerdo a STPP y UNICEF (2014), existen datos que demuestran que los mayores retornos de inversión en las personas se obtienen cuando los recursos que se destinan a las áreas de educación, alimentación adecuada y acceso a salud preventiva y curativa que se dirige a las personas en edad temprana y a los adultos. Se ha valorado que un $40 \%$ de las habilidades cognitivas de la niñez se desarrollan en los primeros 10 años de vida, esto es desde la etapa prenatal. Lo anterior demuestra la validez y la importancia de que las políticas implementadas para solventar estas necesidades de las personas consideren el ciclo de vida de las mismas.

Ahora, si bien estos resultados son importantes, es necesario evidenciar que estos procesos de mejora de las capacidades de las personas no deben ser aislados, y por tanto deben ir acompañados de políticas de Estado que busquen resolver otros problemas estructurales de la economía.

\section{Evidencia empírica global de la descobertura de las dimensiones de la fuerza de trabajo y la pérdida de productividad: salud, educación y nutrición}

En la actualidad diversas instancias reconocen la importancia de la inversión pública en rubros como salud, educación y la garantía del acceso a una buena alimentación, pues la cobertura de tales 
necesidades no solamente implica una cuestión de derechos, sino que, en términos económicos, son un prerrequisito para el desarrollo económico y social.

En definitiva, para alcanzar el desarrollo económico y social es necesario el crecimiento económico, pero eso no es suficiente, pues debe considerarse la calidad del mismo. Al respecto, Garnier (2001) señala la importancia de una política social que garantice el acceso universal a los servicios de educación, salud, vivienda y saneamiento ambiental, ya que son fundamentales para la mejora en la calidad de vida de los trabajadores, como de su capacidad productiva. Para ello es importante que exista un Estado que sea el gestor de la política del desarrollo.

\subsection{Estimaciones del impacto redistributivo de la inversión social y su relación sobre la cobertura de las necesidades en los estratos de menores ingresos}

Con el objetivo de tener un acercamiento del alcance de las políticas sociales sobre los estratos más pobres, a continuación se realiza un análisis sobre la distribución del gasto público social en educación, por quintiles de ingresos de los hogares.
En el cuadro 3 se muestra el porcentaje del gasto público que recibe cada uno de los quintiles de la población en los distintos niveles de enseñanza para el año 2012. Tal como se puede apreciar, los quintiles más pobres son quienes tienen una mayor participación en el gasto público que está destinado a la educación preescolar y primaria, así como secundaria. Por otro lado, quienes tienen un mayor acceso a la educación terciaria son los dos quintiles más ricos, quienes reciben más del $60 \%$ del gasto público que se destina para la educación superior. Es importante mencionar que los recursos destinados a la educación terciaria son mínimos.

Dicho cuadro también muestra las dramáticas desigualdades en la distribución del ingreso. Así, al observar la participación en el ingreso sin Gasto Público Social, se tiene que el quintil más rico de los hogares se apropió de más ingresos que lo que obtuvieron en su conjunto los tres quintiles más pobres. Esto produce que el coeficiente de Gini global sea de 0.41 mientras que al introducir el efecto del GPSE, este se reduce a 0.38, lo cual implica una mínima reducción en la desigualdad del ingreso de 0.03 . 
Cuadro 3. Simulación del efecto redistributivo del Gasto Público Social en educación (GPSE) en los Quintiles de Ingresos de los Hogares, 2012

\begin{tabular}{|l|c|c|c|c|c|c|c|}
\hline \multirow{2}{*}{ GPS en educación } & \multicolumn{7}{|c|}{ Participación (\%) } \\
\cline { 2 - 8 } & Quintil & $\mathbf{1}$ & $\mathbf{2}$ & $\mathbf{3}$ & $\mathbf{4}$ & $\mathbf{5}$ & Total \\
\hline Enseñanza preescolar y primaria & 35.4 & 27.1 & 20.2 & 12.1 & 5.2 & 100 \\
\hline Enseñanza secundaria & 20.7 & 25.0 & 25.9 & 20.5 & 7.9 & 100 \\
\hline Enseñanza terciaria & 2.7 & 9.1 & 23.0 & 33.6 & 31.6 & 100 \\
\hline Efecto redistributivo & 7.0 & 12.2 & 16.6 & 22.3 & 41.9 & 100 \\
\hline Sin GPS en educación & 8.6 & 13.1 & 16.9 & 21.8 & 39.6 & 100 \\
\hline Con GPS en educación & 1.6 & 0.9 & 0.3 & -0.5 & -2.3 & \\
\hline Efecto neto en el ingreso de los hogares & \multicolumn{7}{|c|}{} \\
\hline Efecto en el Coeficiente de Gini & 0.41 & & & & & \\
\hline Sin GPS en educación & 0.38 & & & & & \\
\hline Con GPS en educación & 0.03 & & & & & \\
\hline Reducción en Coeficiente de Gini & & & & & \\
\hline
\end{tabular}

Fuente: Elaboración propia con base en datos de la Encuesta de Hogares y Propósitos Múltiples (EHPM), 2012, y la Secretaría Técnica y de Planificación de la Presidencia de la Presidencia (STPP), 2014.

Además, es importante conocer que la carga tributaria posee una estructura altamente regresiva, donde en el 2006 el decil más pobre pagaba 3 veces más impuestos que el decil más rico, en relación con sus ingresos (ICEFI-BID, citado en Ministerio de Hacienda, 2010). Con las reformas fiscales a partir del 2009, probablemente esta estructura regresiva se redujo. A la información anterior hay que agregar el hecho que, a pesar que los recursos destinados a educación representan una porción importante del presupuesto total y que la estructura de gasto es progresiva, el gasto en educación no revierte la distribución desigual del ingreso.
De esta forma existe evidencia que la baja cobertura de la fuerza de trabajo se explica, en gran parte, por la gran desigualdad que impera en el país, pues muy pocas personas concentran los principales medios de producción en el país. Lo anterior permite sostener que aún hay espacios para realizar una reforma fiscal que lleve a una carga tributaria más progresiva y que permita incrementar el gasto social, o incluso, que se lleven a cabo políticas económicas estructurales que mejoren la distribución primaria del ingreso de los hogares, como por ejemplo, una política de incremento de salarios, entre otras medidas. 


\subsection{Evidencia empírica global}

\subsubsection{Enfoque del desarrollo humano}

Ciertamente existe una estrecha relación entre crecimiento económico y mejoras en la calidad de vida de las personas, entendida esta como desarrollo humano. Pero hay que analizar detenidamente cuál de los dos factores debe garantizarse primero. De acuerdo con Ranis y Stewart (2002):

\begin{abstract}
No hay duda alguna de que existe una firme relación recíproca entre ambos. Por una parte, el crecimiento económico proporciona los recursos que permiten mejorar ininterrumpidamente el desarrollo humano; por la otra, el mejoramiento de la calidad de la mano de obra contribuye de manera importante al crecimiento económico. (p. 8)
\end{abstract}

Resulta claro que desarrollo humano y crecimiento económico presentan una estrecha vinculación en doble vía, pues numerosos estudios han demostrado las pérdidas económicas que se derivan de factores asociados a la descobertura de salud, educación y alimentación adecuada.

Rivera y Currais citado en Ávila \& Briseño (2008) sostienen que la correlación entre mejor salud y crecimiento económico se mantiene aun cuando se introducen otro tipo de variables. Su estimación indica que cada mejora de un $10 \%$ en la esperanza de vida al nacer, se asocia a un aumento del crecimiento económico anual de al menos 0.3 a 0.4 puntos porcentuales.

\subsection{Costos económicos por la insuficiente cobertura de la dimensiones de la fuerza de trabajo}

\subsubsection{Salud}

Estudios de la OPS y CEPAL muestran que en Iberoamérica existe una fuerte relación entre la esperanza de vida y la tasa de crecimiento del PIB: los resultados sugieren que por un año adicional de esperanza de vida, habría un aumento adicional de $1 \%$ en el PIB quince años después (Garnier, 2000). Asimismo, investigaciones recientes sugieren que en los países pobres, las inversiones en salud sobre el crecimiento económico poseen un impacto relevante. Bhargava, Jamison, Lau y Murray (2000), mostraron efectos significativos de la tasa de supervivencia adulta (TSA) sobre el crecimiento económico, determinando que para los países de menor renta, una variación positiva de $1 \%$ en TSA está asociado con un aumento de $0.05 \%$ en la tasa de crecimiento, señalando además que, si bien parece un impacto bastante bajo, 
es mucho mayor que el impacto de $0.014 \%$ que se obtiene al aumentar en $1 \%$ la tasa de inversión respecto al PIB.

Dentro de las dimensiones que conforman el ser humano, la salud parece ser una de las más obvias para garantizar que las personas generen el mayor rendimiento dentro de los trabajos que desempeñan. Las enfermedades claramente perjudican las condiciones humanas, no solo por las horas perdidas ante los días de incapacidad que generan en los trabajadores y trabajadoras, sino que incluso puede conllevar a la muerte misma de las personas y por lo tanto a la pérdida total de la fuerza productiva más importante que posee una sociedad.

Resulta, por lo tanto, de vital importancia el papel que pueda desempeñar la inversión social para garantizar la cobertura del valor de la fuerza de trabajo. Una vez expuesto lo anterior, es claro que no se trata de que el gobierno financie cualquier tipo de bienes o invierta en cualquier tipo de factor productivo, sino que se trata de identificar y reconocer la importancia de la fuerza de trabajo y su calidad de ser humano para brindarle a la misma las condiciones de vida digna que necesita, lo cual claramente terminará redundando en mayores beneficios productivos para toda la sociedad.

\subsubsection{Educación}

La evidencia estadística indica que el nivel de educación alcanzado es un factor importante para explicar las diferencias de ingreso en el mercado laboral. Por ejemplo, estimaciones realizadas en varios países de América Latina demuestran que las personas que pasan el umbral de 12 años de educación tienen mayor probabilidad de acceder al bienestar (CEPAL, 2012). De esta forma, la meta de educación media completa para toda la población, para los países subdesarrollados, se transforma en un requisito básico de la oferta de fuerza de trabajo para alcanzar el bienestar de la sociedad.

Siguiendo con la investigación de la CEPAL (2010), se estima que cada año adicional de escolaridad está asociado a un aumento de 0.58 puntos porcentuales de crecimiento de largo plazo, los resultados macroeconómicos más confiables son aquellos que proveen resultados consistentes con las estimaciones microeconómicas de tasas de retorno a la educación (6 a 12\%). Marroquín (2006) realizó un modelo econométrico estimado para Latinoamérica, el cual demostró que el capital humano contribuyó al crecimiento económico en un $5 \%$ por cada $1 \%$ que se incrementó durante el período 1980-2003. Por su parte, las estimaciones realizadas para los países 
miembros de la Organización para la Cooperación y el Desarrollo Económico (OCDE), el capital humano aportó al crecimiento económico un $14 \%$ por cada $1 \%$ que se incrementó el producto en el mismo período. De acuerdo con la investigación, los 9 puntos porcentuales de diferencia en el aporte al crecimiento por parte de capital humano al crecimiento económico vienen explicados por el rezago en la cantidad y calidad del mismo.

De este apartado se concluye que la mejora en educación posee impactos positivos en la economía e incluso en el crecimiento económico, lo que ayuda a eliminar la noción que el gasto público en educación es una carga que debe ser atendida luego de alcanzar el crecimiento económico. $Y$ es que resulta claro que aspectos como la oferta académica, tasa de analfabetismo, tasa de matriculación primaria y gasto público, terminan impactando en el crecimiento económico vía aporte de la fuerza de trabajo capacitada, instruida y educada.

Para ser más precisos, hay estudios como el de McMahon citado en CEPAL, (2010), que muestran que los impactos de la educación no se limitan al crecimiento económico, sino que también existe un claro efecto sobre variables claves del desarrollo. Así por ejemplo, con relación al impacto económico, una mayor educación garantiza una mayor estabilidad en el proceso productivo y permite una mayor tasa en la inversión en capital fijo; contribuye a desarrollar la investigación, desarrollo e innovación permitiendo a su vez, la creación de nueva tecnología, entre otros efectos. Por el lado de los impactos sobre el desarrollo, una mayor educación tiene la capacidad de mejorar la salud pública, mejora los procesos de democratización (debido a la mayor alfabetización, entre otros factores), brinda una mayor estabilidad política al mejorar el funcionamiento de las instituciones; genera mayor conciencia sobre la importancia de los derechos humanos, disminuye la propensión al crimen, disminuye la deforestación, y reduce la pobreza, la desigualdad y la exclusión social, entre otros factores.

Sin embargo, se debe reconocer que no basta con destinar recursos para la educación si no se posee una estrategia definida. Es importante que el Estado determine el tipo de educación que se requiere e identifique mecanismos que mejoren la calidad educativa en el país, pues de esto depende el aporte de la educación sobre el crecimiento económico. Además, también es claro que para el caso de las mujeres el valor de la fuerza de trabajo no es valorado debido a motivos subjetivos. Esto muestra que las medidas enfocadas en asegurar la cobertura del valor de la fuerza de trabajo deben elaborarse de 
manera integral, de modo que se garantice que no ocurran este tipo de discriminaciones.

Por último, se reconoce que la inversión en educación debe ser complementada con cambios en la estructura económica (mercado laboral, etc.) de modo que la fuerza de trabajo sea valorada de mejor manera por los capitalistas.

\subsubsection{Nutrición}

De acuerdo con la FAO (2013), el costo social de la malnutrición -medido por los años de vida perdidos a causa de la malnutrición infantil y materna- del sobrepeso y la obesidad, es muy alto. En gran medida esto se debe a que los problemas de desnutrición también impactan en otras dimensiones de la vida humana, pues el retardo de crecimiento que se deriva "está asociado con una reducción substancial en la capacidad mental y con un rendimiento escolar deficiente, lo cual finalmente conduce a una productividad laboral reducida" (FAO, 2013).

El mismo documento señala que, de acuerdo al Programa Mundial de Alimentos, cada año el costo de hacerle frente al problema de la desnutrición en niños y niñas menores de 5 años, equivale a 2.05 mil millones de dólares, un costo bastante bajo, si se tiene en cuenta que el costo de no combatirla oscila entre 104 y 174 mil millones de dólares; por mortalidad infantil, pérdida en la productividad por retardo en el crecimiento y pérdidas por enfermedades crónicas.

A continuación se presentan una serie de estimaciones propias de los costos que generan algunas problemáticas sociales:

\subsubsection{Costo económico de la repitencia estudiantil asociada a enfermedades}

Dado que la inversión en salud disminuye la probabilidad de contraer enfermedades, esto genera efectos positivos en otras dimensiones de la fuerza de trabajo. Dentro del área de educación el padecimiento de enfermedades es una de las principales causas de la repitencia y abandono estudiantil, por lo que es importante valorar y realizar una estimación de los costos económicos que generan los problemas de salud en educación. Es decir, se plantea que es más eficiente invertir en salud para ahorrarse los costos económicos de la repitencia estudiantil, para lo cual son necesarias establecer políticas de prevención de enfermedades. En el siguiente cuadro se presentan los resultados de las estimaciones realizadas. 


\section{Cuadro 4. Costo de repitencia escolar por problemas de salud y discapacidad, 2012}

\begin{tabular}{|l|c|c|}
\cline { 2 - 3 } \multicolumn{1}{c|}{} & \multicolumn{2}{c|}{ Nivel educacional } \\
\cline { 2 - 3 } \multicolumn{1}{c|}{} & Básico & Medio \\
\hline Número alumnos por nivel repitiendo grado & 8,868 & 169 \\
\hline Costo anual por estudiante (USD 2012) & 464.7 & 410.8 \\
\hline \multirow{2}{*}{ Costo anual adicional (USD 2012) } & $4,121,027.3$ & $69,428.5$ \\
\cline { 2 - 3 } & \multicolumn{2}{|c|}{$4,190,455.7$} \\
\hline Porcentaje gasto educación & \multicolumn{2}{|c|}{$0.56 \%$} \\
\hline Porcentaje gasto salud & \multicolumn{2}{c|}{$1.74 \%$} \\
\hline Porcentaje PIB & \multicolumn{2}{|c|}{$0.02 \%$} \\
\hline
\end{tabular}

Fuente: Elaboración propia con base en datos de EHPM 2012, y en CEPAL, 2007.

3.3.5 Costo económico de la repitencia estudiantil asociada a problemas económicos de hogares:

Un significativo porcentaje de alumnos que han estado inscritos repiten grado debido a problemas económicos y/o a la necesidad de trabajar. Si se parte que la fuerza de trabajo debe asegurar la cobertura de sus necesidades y por ende su reproducción, esto no debe enfrentar limitantes si su deseo es estudiar, y más bien debe recibir incentivos para ello.

Por tanto, la ocurrencia de la repitencia estudiantil por problemas económicos implica cierta responsabilidad del gobierno, ya que al no evitar esta situación incurre en costos económicos extras. La idea es garantizar que los individuos accedan (y tengan incentivos para acceder) al sistema educativo, sin enfrentar la disyuntiva de abandonar o no los estudios.

\section{Cuadro 5. Costo de repitencia escolar por problemas económicos y por necesidad de buscar empleo, 2012}

\begin{tabular}{|l|c|c|}
\cline { 2 - 3 } \multicolumn{1}{c|}{} & \multicolumn{2}{c|}{ Nivel educacional } \\
\cline { 2 - 3 } \multicolumn{1}{c|}{} & Básico & Medio \\
\hline Número alumnos por nivel repitiendo grado & 4,410 & 343 \\
\hline Costo anual por estudiante (USD 2012) & 464.7 & $140,911.1$ \\
\hline \multirow{2}{*}{ Costo anual adicional (USD 2012) } & $2,049,360.6$ \\
\cline { 2 - 3 } & \multicolumn{2}{|c|}{$2,190,271.7$} \\
\hline Porcentaje gasto educación & \multicolumn{2}{|c|}{$0.29 \%$} \\
\hline Porcentaje PIB & \multicolumn{2}{|c|}{$0.01 \%$} \\
\hline
\end{tabular}

Fuente: Elaboración propia con base en datos de EHPM, 2012 y en CEPAL, 2006. 


\subsubsection{Estimación de pérdida de productividad por desnutrición crónica}

De acuerdo a la CEPAL (2006), la desnutrición afecta negativamente a la productividad porque la población desnutrida alcanza menores niveles de escolaridad, y a su vez la mayor probabilidad de muerte de este grupo poblacional implica horas de trabajo perdidas.

En el presente apartado se hará una aproximación a los niveles de productividad perdida por la mortalidad asociada a la desnutrición, siguiendo la metodología aplicada en el estudio "El Costo del Hambre" por la CEPAL en 2007, por lo que se tomó a la población infantil de 0 a 5 años (período 2010-2014), y se consideró la tasa de mortalidad infantil y de prevalencia de la desnutrición infantil. Además, entre otros supuestos, se consideró que para la población desnutrida la tasa de mortalidad infantil se mantiene al mismo nivel que en la población no desnutrida. Este supuesto tiende a subvaluar la mortalidad infantil asociada a la desnutrición. Por último, se estimó la productividad perdida durante toda la etapa productiva de la población que se consideró que en este caso abarcaría desde el año 2024 a los 2078 (54 años), pues hasta el 2024 la población de 0 a 5 años llegaría a la edad en que se permite trabajar.

\section{Cuadro 6. Pérdida de productividad debido a la mortalidad infantil asociada a la desnutrición, 2012}

\begin{tabular}{|l|r|}
\hline Nacimientos quinquenio 2010-2014 & 623,230 \\
\hline Tasa de mortalidad infantil (0-5 años) (por 1000 nacidos vivos) & 19 \\
\hline Desnutrición crónica & 19.2 \\
\hline Niños y niñas con enfermedades relacionadas a desnutrición & 119,660 \\
\hline Mortalidad infantil asociada con desnutrición crónica & 2,274 \\
\hline costo millones de mortalidad (USD millones) & 12.7 \\
\hline Porcentaje de PIB & $0.05 \%$ \\
\hline
\end{tabular}

Fuente: Elaboración propia con base en datos de CELADE - División de Población de la CEPAL. Revisión 2013, y en CEPAL (2008).

De lo planteado en este capítulo se desprende que la búsqueda de soluciones para estos problemas no es solamente de una cuestión de derechos, sino que la ampliación universal de los servicios sociales básicos debe estar fundamentada en decisiones de política y sus impactos en la estructura económica, y además teniendo en cuenta los costos económicos que asume el conjunto de la sociedad por no atender a tiempo las distintas problemáticas sociales. 


\section{Logros y desafíos de los programas sociales: propuestas y recomendaciones para la mejora de la inversión pública en salud y educación}

\subsection{Avances y limitaciones del sistema de protección social universal}

Una de las acciones concretas que ha implementado el último gobierno para mejorar las condiciones de las personas es el SPSU. Respecto a este último, ya se ha citado que es el enfoque del ciclo de vida y de derechos de los fundamentos teóricos que lo sustentan.

Al contrastar el papel que ha logrado desempeñar el SPSU y lo que plantea el enfoque del ciclo de vida, se puede concluir que la gestión del gobierno comprendido entre 2009 y 2014 obtuvo importantes avances. Sin embargo, pese a la existencia de los programas antes mencionados, existe una gran deuda por parte del SPSU para cubrir las necesidades que las personas experimentan en sus primeros años de vida. Lo anterior debido a que el sistema es en realidad adulto céntrico, destinando así la mayor parte de recursos a esta parte de la población.

Por otro lado, la existencia del enfoque de derechos en la formulación de la política social en el caso de El Salvador ha permitido generar las condiciones para crear instrumentos legales que comprometan al
Estado a atender las necesidades de la población dentro de los límites que le permiten sus finanzas, un claro ejemplo de esto fue la aprobación de la Ley de Desarrollo y Protección Social.

Sin embargo, a pesar de la concretización de la Ley, el Estado sigue teniendo una gran deuda, en cuanto a la universalidad y participación de los programas del SPSU, pues pocos de ellos permiten entrelazar la participación de las personas hacia las cuales se dirigen, y dada la situación financiera que enfrenta el país, el principio de la universalidad es el que parece cumplirse con menor cabalidad.

Ahora bien, existen otros problemas que obstaculizan la eficiente implementación de la política social en El Salvador: en primer lugar la existencia de un alto porcentaje de analfabetas funcionales, que no solo están invisibilizados en las investigaciones estadísticas, sino que además, al limitar las capacidades de las personas, se transforma en un círculo vicioso que no permite el correcto desarrollo de los programas sociales. Este fenómeno podría corregirse con mayores esfuerzos por parte del Estado si se lograrán solventar las necesidades de la primera infancia. 
En segundo lugar, existen debilidades por parte de los hacedores de política, y es que como primer punto se puede mencionar que en STPP, existe poco personal con conocimientos acerca de seguridad social, lo cual dificulta el proceso de creación y preparación de políticas destinadas a esta área. Además, se evidencia una falta de planificación y formalidad en los estudios previos que se requieren para llevar a cabo dichas políticas y existe una falta de documentación recopilada por la misma institución para la planificación de los programas sociales, que no solo dificulta las investigaciones que quieran llevarse a cabo respecto a los programas que implementan, sino que genera una pérdida de continuidad en los procesos de implementación y planificación, y esto finalmente produce resultados menos eficientes.

$Y$ como tercera falencia, se observa que pese a que el enfoque bajo el cual se fundamentan los programas sociales ya no es asistencialista, aún es posible observar en la mayoría de ellos que la capacitación y la ayuda que se otorga a las personas sigue sin involucrar la participación de las mismas.

Los programas gubernamentales deben ir orientados a propiciar procesos inclusivos, participativos, sostenibles, componibles, legítimos, etc. En este sentido, al analizar los programas llevados a cabo en el periodo 2009-2014, se deduce que a pesar que el sistema ha realizado importantes esfuerzos para elevar las condiciones de vida de la población no existe una estrategia o programa cuya lógica y objetivo sea asegurar la reproducción de la fuerza de trabajo.

\subsection{Comparación entre los logros de los programas gubernamentales (2009-2014) y la propuesta de Max Neef}

Al evaluar los diferentes programas gubernamentales 20092014, a la luz de la propuesta de Max Neef (1993), se pretende verificar la compatibilidad de estos con la concepción de los satisfactores, ya que estos últimos son los que suponen dar cobertura al valor de la fuerza de trabajo, especialmente aquellos que son sinérgicos.

Los programas que han sido identificados con algún grado de sinergia, es decir, con capacidad de no solo satisfacer la necesidad para la cual fueron creados, sino que además estimular la satisfacción de otras necesidades, fueron: El programa de dotación de uniformes y útiles escolares, el programa "Vaso de leche", el Programa de Agricultura Familiar (PAF) y los Equipos Comunitarios de Salud Familiar, algunos con mayor o menor grado de sinergia que se detalla a continuación: 
El programa de dotación de uniformes y útiles escolares, además de haber incrementado la asistencia escolar se consideró como un satisfactor sinérgico ya que en un principio el programa pretende cubrir una necesidad de subsistencia, sin embargo, al mismo tiempo estimula de gran manera la participación de los proveedores y su crecimiento empresarial, al igual que el programa "Vaso de leche" que además de asegurar la nutrición de los estudiantes estimula la producción nacional y el desarrollo ganadero mediante estas políticas. Sin embargo, para que estos tengan un mayor grado de sinergia, es necesario que se incremente la participación por parte de todos los actores, ya que según las evaluaciones realizadas, las instituciones de gobierno y las familias son quienes tienen menor calificación en la participación.

El Programa de Agricultura Familiar (PAF), y el de Equipos Comunitarios de Salud Familiar (ECOS), son los que han evidenciado tener un mayor involucramiento por parte de las comunidades, propiciando así un mayor alcance de necesidades, ya que la participación y organización son fundamentales para que estos programas sean considerados sinérgicos.
Mientras que otros programas como el PATI y Nuestros Mayores Derechos, al consistir en la entrega de una ayuda monetaria, no propician la estimulación hacia la satisfacción de otras necesidades y no han sido considerados como sinérgicos, al igual que el programa de Comunidades Solidarias, pues no existe una participación por parte de las personas que les permita incidir directamente sobre la orientación del proyecto, ni se recibe una retroalimentación de los programas por parte de la comunidad.

De estos programas, y a partir de la propuesta de Max Neef, se evidencia que es necesario romper con la dependencia de los programas asistenciales y promover la auto dependencia que según Max Neef (1993) genera efectos sinérgicos y multiplicadores, porque van de "abajo hacia arriba". Así se entiende que son los espacios comunitarios los que permiten potenciar el desarrollo de los territorios, por lo que se necesitan políticas alternativas que busquen estimular la participación y organización de sujetos sociales capaces de ser los propios autores de un desarrollo autónomo, auto sustentado e integral, y de esta forma garantizar la sostenibilidad de los proyectos. 


\subsection{Propuesta de mejora de políticas públicas desde el enfoque de la teoría del valor de la fuerza de trabajo}

\subsubsection{Alimentación}

Ya se ha demostrado a lo largo de la presente investigación la incidencia que una buena nutrición tiene en la productividad individual y agregada de los trabajadores, y si bien la alimentación es solo una proporción del valor de la fuerza de trabajo, es una de las que deben cubrirse desde la concepción de la vida, ya que esta condiciona o condicionará en gran medida las capacidades humanas y productivas. Es por esto que se considera necesario proponer una serie de medidas que permitan que la CBA, más allá de ser un mero instrumento de medición de pobreza o como referente de los requerimientos mínimos calóricos, se convierta en un instrumento de política pública que involucre a diversos sectores de la población, y cuya estructuración rompa con el esquema de verticalidad.

Se propone la conformación de canastas alimentarias que atiendan al ciclo de vida, es decir que la configuración de la misma atienda a diferentes grupos etarios o etapas de vida, pues tanto la actual CBA como la CBA propuesta (abordada en el capítulo II), están pensadas para un adulto promedio. Sin embargo, en niños y niñas menores de 5 años, mujeres embarazadas y lactantes, $\mathrm{u}$ otros grupos poblacionales, los requerimientos de ingesta alimentaria son obviamente diferentes al del patrón típico. Además, se propone que estas canastas se basen en patrones de consumo actualizados, ya que existe un desfase de 8 años (como la CBA propuesta, basada en la Encuesta Nacional de Ingresos y Gastos de los Hogares 2006 de México), que puede significar una lectura errónea de lo que realmente se requiere en la actualidad.

Por otro lado, para garantizar un mayor acceso a los alimentos y aseguramiento de los mismos durante todo el año, se propone la conformación de canastas alimentarias temporales, es decir que contemplen en su composición alimentos sustitutivos, de acuerdo al ciclo de producción, y esto a su vez contribuiría a una dieta más diversa. Para ello es necesario un esfuerzo del Estado que identifique qué alimentos se producen, en qué tiempos y a cuáles sustituyen.

Asimismo, se propone que la canasta básica alimentaria sea creada teniendo en cuenta la posibilidad de promover mercados locales, de tal forma que esté constituida por alimentos que además de ser nutricionalmente adecuados, sean de carácter endémico, la conformación de mercados locales o redes de abasto que garanticen el acceso físico a los alimentos. Esto 
puede contribuir también a incentivar la producción en los territorios, disminución de los precios (vía disminución de costos de transporte), y surgimiento de un círculo virtuoso que ayude a las personas a superar el umbral de la pobreza (los productores locales por ejemplo).

\subsubsection{Democracia Económica}

A partir de esta investigación se propone que la forma de gestión económica-empresarial en El Salvador debe conciliar las voluntades de los capitalistas con los de la fuerza de trabajo, principalmente mediante una institucionalidad que posea un amplio respaldo ciudadano, lo que podría mejorar la democracia económica del país.

Para ello se propone concretamente:

- La creación de instrumentos legales que garanticen que los trabajadores, como únicos generadores de valor, no sean excluidos en la toma de decisiones de las unidades productivas en donde ellos operan, de tal manera que en las instancias en donde se tomen las decisiones exista una igualdad de representantes de los trabajadores y de los capitalistas.

- Que se incluya en este modelo de cogestión a representantes de la sociedad civil organizada en las decisiones empresariales que tengan impactos externos a la empresa.

- El derecho a veto de los trabajadores, este para la toma de aquellas decisiones que puedan representar efectos adversos en la reproducción de la fuerza de trabajo.

- Incentivar la creación de cooperativas y asociaciones productivas y la creación de redes para potenciar su producción y comercio, con un acompañamiento estatal, especialmente en el tema de creación de créditos estatales para la modernización de las unidades productivas de estas cooperativas.

- Instrumentos tributarios y fiscales progresivos.

\subsubsection{Organización y participación comunitaria}

Una de las características de las administraciones actuales es que diseñan y ejecutan las políticas sociales sin tomar como punto de partida las necesidades de las personas hacia las cuales están dirigidas. Ante ello, resulta necesaria una reestructuración en la administración gubernamental que permita involucrar dichos intereses colectivos. Por lo que se recomienda, entre otras cosas, descentralizar el proceso que se lleva a cabo para la planificación e implementación 
de las políticas sociales a través de una mayor participación de los gobiernos locales como ejes de desarrollo territoriales, ya que son estos quienes tienen la posibilidad de acercarse a la población hacia la cual se dirigen.

En este sentido, tanto la organización como la participación de las comunidades, resulta fundamental no solo para garantizar la sostenibilidad de cualquier tipo de programa gubernamental o no gubernamental, sino que también para lograr un verdadero desarrollo en los territorios. Es decir, se propone romper con los esquemas de dependencia y asistencialismo, y que se transformen los proyectos para que las comunidades sean autogestionarias de su propio desarrollo.

Desde esta perspectiva resulta que el papel que debe jugar el Estado es de promover procesos sinérgicos, en tanto que posibiliten la satisfacción simultánea de otras necesidades dentro de las comunidades. Además, el Estado debe ser un facilitador de los procesos y no entorpecer el trabajo de las mismas, en el sentido de que la asistencia profesional y técnica que se ofrezca debe atender a la lógica de organización de estos colectivos.

Finalmente, al otorgar un mayor peso a las municipalidades, se espera que estas tengan un papel más activo en la compilación de información respecto a los proyectos que se ejecutan en sus localidades. Generalmente la información estadística y cualitativa respecto a las políticas sociales ejecutadas está disponible a nivel departamental, lo cual impide conocer el verdadero alcance que tienen los proyectos en los territorios más pequeños y alejados.

\section{Conclusiones}

La participación del Estado en el proceso de política social es fundamental, lo cual queda claro, pero lo que no es evidente es que estos procesos de participación deben ser integrados en un consenso social, en el cual no intervenga únicamente el Estado, sino además, empresarios y trabajadores. La participación de los trabajadores en las decisiones empresariales no es una práctica generalizada, y menos aún en economías subdesarrolladas como la salvadoreña.

Se ha logrado concluir que es esencial encaminarse a un modelo que garantice que la capacidad de influencia de los trabajadores dentro de las decisiones productivas empresariales sea real, de tal manera que las empresas guíen sus decisiones de acuerdo a las necesidades de la sociedad en su conjunto, y el Estado por su parte, 
debe acompañar el proceso de cogestión mediante el establecimiento de leyes y fortalecimiento de la institucionalidad, y con ello garantizar la reproducción de la fuerza de trabajo.

Además, estos procesos de inversión social no deben ser aislados, y por tanto deben ir acompañados de políticas de Estado que busquen resolver otros problemas estructurales del país. Ya se ha evidenciado que a pesar que se logren efectos positivos, si estos no van acompañados de mejoras en la economía en general, estos resultados serán mermados.

En este sentido, dado que se está hablando de una mejora en las capacidades y potencialidades de las personas, si se posee un mercado laboral deficiente y una economía desarticulada, esto no se traducirá en un pleno aprovechamiento.

Finalmente, una de las conclusiones más fundamentales es que el desarrollo no debe entenderse como crecimiento, es algo más complejo que involucra capacidades, participación, libertad, condiciones de subsistencia y laborales adecuadas, consenso social etc., donde el funcionamiento normal de la fuerza de trabajo y el correspondiente aprovechamiento tecnológico en el proceso productivo juegan un papel determinante. De esta manera, al concluir que la fuerza de trabajo es la fuerza productiva más importante de una sociedad, se sugiere inevitablemente que una cobertura insuficiente impactará negativamente en su desempeño y en su economía, obstaculizando así un pleno desarrollo.

En otras palabras, en la presente investigación se constata que de no cubrirse las tres necesidades básicas abordadas en el estudio: alimentación, salud y educación, se generará una cadena de efectos negativos en la economía que más tarde o más temprano comprometerá la fuerza laboral del futuro. 


\section{Referencias}

- Ávila, N. \& Briseño, W. (2008). "Inversión en la salud-crecimiento económico y desarrollo humano en la República Bolivariana de Venezuela. Período 1990-2008". Negotium, 7 (11), 126-161.

- Bhargava, A., Jamison, D. T., Lau, L. J. y Murray, C. J. L. (2000). "ModeIling the Effects of Health on Economic Growth". Ginebra: Organización Mundial de la Salud (Global Programme on Evidence Discussion Paper).

- Castaneda, I. y Rivera J. (2010). Pobreza extrema y política nacional de seguridad alimentaria y nutricional en El Salvador (2000-2008). (Tesis de pregrado). UCA. El Salvador.

- Comisión Económica para América Latina y el Caribe, CEPAL. (2006). Boletín de la infancia y adolescencia sobre el avance de los objetivos de desarrollo del Milenio.. Santiago de Chile.

- Comisión Económica para América Latina y el Caribe, CEPAL. (2010) Panorama social de América Latina 2010. Santiago de Chile. Recuperado de http://www.eclac.org/publicaciones/xml/9/41799/PSE2010-Cap-IIeducacion-preliminar.pdf

- Comisión Económica para América Latina y el Caribe, CEPAL. (2012). Panorama Social 2012. Santiago de Chile. Recuperado de http://www. cepal.org/cgi-bin/getProd.asp?xml=/publicaciones/xml/5/48455/P48455. $\mathrm{xml} \& \mathrm{xs}=/ \mathrm{tpl} / \mathrm{p} 9 \mathrm{f} . \mathrm{xs} \mid \&$ base=/tpl/top-bottom.xsl\#

- Organización de las Naciones Unidas para la Agricultura y la Alimentación, FAO. (2000). Estado Mundial de la Agricultura y la alimentación. Recuperado de http://www.fao.org/docrep/017/x4400s/x4400s.pdf

- Organización de las Naciones Unidas para la Agricultura y la Alimentación, FAO. (2013). Estado Mundial de la Agricultura y la Alimentación. Recuperado de www.fao.org/publications/sofa/es/

- Organización de las Naciones Unidas para la Agricultura y la Alimentación, FAO. (2010). "Folleto formativo №34: El Derecho a la Alimentación Adecuada". Recuperado de http://www.ohchr.org/Documents/ Publications/FactSheet34sp.pdf

- Garnier, L. (2000). La Inversión Social y el Costo De Las Oportunidades Pérdidas. CEPAL-UNICEF-SECIB. Santiago de Chile. Recuperado de http://www.oei.es/observatorio2/cepal05.pdf

- Marroquín, E. (2006). Análisis Del Capital Humano, Productividad Y Crecimiento Económico, Desde La Perspectiva De La Función De Producción. Universidad de San Carlos de Guatemala. 
- Max Neef, M. (1993). Desarrollo a Escala Humana: Conceptos, aplicaciones y algunas reflexiones. Montevideo, Uruguay: Editorial Nordan-Comunidad.

- Ministerio de Hacienda. (1995-2001). Informe Complementario Constitucional de la Hacienda Pública. Gobierno de El Salvador.

- Ministerio de Hacienda. (2002-2012). Resumen Ejecutivo: Ejecución Presupuestaria de Gastos. Gobierno de El Salvador.

- Ministerio de Hacienda. (2012). Boletín Presupuestario (Enero-Marzo 2012). Gasto público en educación en El Salvador, periodo 2000-2012. Dirección General de Presupuesto. Recuperado de http://www.mh.gob. sv/portal/page/portal/PCC/Boletin2012/Boletin_Presupuestario_AbrilJunio_2012.pdf

- Montesino, M. (1998). Efecto de la dinámica salarios-productividad en el desarrollo económico. Caso El Salvador (1974-1995). (Tesis de Maestría en Economía y Desarrollo) Postgrado Centroamericano en Economía y Planificación del Desarrollo. Universidad Nacional Autónoma de Honduras.

- Montesino, M. (2000). "La dinámica salarios-productividad y el desarrollo económico en El Salvador". Realidad, Revista de Ciencias Sociales y Humanidades, 76, 377-407.

- Montesino, M. (2007). Importancia del valor de la fuerza de trabajo y de la racionalidad reproductiva para la gestión del desarrollo. Disertación por el Doctorado en Gestión de desarrollo. Universidad Nacional Autónoma de Honduras.

- Montesino, M. (2011a). Contribución a la teoría del desarrollo de las formaciones socioeconómicas de Carlos Marx: complejidad y carácter general. San Salvador: UCA Editores.

- Montesino, M. (2011b). Economía y desarrollo: racionalidad reproductiva y valor de la fuerza de trabajo en la gestión del desarrollo. San Salvador: UCA Editores.

- Montoya, A. (2000). Apuntes de Desarrollo Económico. San Salvador: Editores Críticos.

- Programa de las Naciones Unidas para el Desarrollo, PNUD. (2007). Operacionalización de los enfoques basados en los derechos humanos para la reducción de la pobreza. Informe provisorio sobre un proyecto piloto. Nueva York. Recuperado de: http://www.undp.org/content/undp/ es/home/librarypage/poverty-reduction/los-enfoques-basados-en-losderechos-humanos-para-la-reduccin-de-la-pobreza.html 
- Programa de las Naciones Unidas para el Desarrollo, PNUD (2013). Informe sobre Desarrollo Humano El Salvador 2013. Imaginar un nuevo país. Hacerlo posible. Diagnóstico y propuesta. San Salvador.

- Ranis, G. y Stewart, F. (2002). "Crecimiento y Desarrollo Humano en América Latina". Revista CEPAL, 78, 7-24.

- Secretaría Técnica y de Planificación de la Presidencia de la Presidencia, STPP. (2014). Gasto Público Social. Unidad de Evaluación de Políticas y Programas. Mimeo. San Salvador.

- Secretaría Técnica y de Planificación de la Presidencia de la Presidencia - Fondo de las Naciones Unidas para la Infancia - Instituto Centroamericano de Estudios Fiscales, STPP-UNICEF-ICEFI. (2014). El Salvador: Medición del gasto público en niñez y adolescencia 2011. San Salvador.

- Sen, A. (2000). Desarrollo y libertad. Barcelona, España: Editorial Planeta. 